\title{
Shedding light on the graph schema: Perceptual features versus invariant structure
}

\author{
RAJ M. RATWANI \\ George Mason University, Fairfax, Virginia \\ AND \\ J. Gregory Trafton \\ Naval Research Laboratory, Washington, D.C.
}

\begin{abstract}
Most theories of graph comprehension posit the existence of a graph schema to account for people's prior knowledge of how to understand different graph types. The graph schema is, however, a purely theoretical construct: No empirical studies have explicitly examined the nature of the graph schema. We sought to determine whether graph schemas are based on perceptual features or on a common invariant structure shared between certain graph types. We isolated the process of activating the graph schema by presenting the graphs to participants in pure and mixed blocks. Any differences in reaction time between the blocks could be attributed to loading the appropriate schema. Results from a series of experiments using five types of graphs suggest that graph schemas are based on the graphical framework, a common invariant structure among certain types of graphs. These results provide insight into the comprehension of novel graphs.
\end{abstract}

Graphs provide useful and efficient ways in which to display information. When asked to extract information from a graph, people generally have some stored knowledge that they use to comprehend the graph, despite the fact that different graph types represent information differently. How do theories of graph comprehension account for our prior knowledge of how to interpret different graphs?

By focusing on how specific information is extracted from a graph, researchers have developed several theories of graph comprehension (Lewandowsky \& Behrens, 1999; Lohse, 1993; Peebles \& Cheng, 2001, 2003; Pinker, 1990; Shah \& Carpenter, 1995; Simkin \& Hastie, 1987). Collectively, these theories suggest the following processes: (1) Construct relationships among the graph elements using early visual processes, (2) build a propositional representation, (3) activate the graph schema, (4) devise the conceptual question (determine desired information), and (5) devise the conceptual message (the answer). Activation of the graph schema (Step 3) is the critical process in accounting for the prior knowledge and specific operations used to comprehend the graph. The schema is a mental representation in long-term memory, activated by matching the early visual input to a stored mental representation used to interpret the graph.

Although researchers agree on the existence of the graph schema as a construct, there is little to no agreement on how it is organized, and there is little to no empirical evidence regarding the defining features of the schema. Such empirical research is lacking partly because there has been no strong methodology with which to examine such a construct. The goal of this study is to begin to understand the features that underlie the graph schema and to illustrate a methodology that can be used to understand schemas in other domains.

\section{Differing Views of the Graph Schema}

Although there is no consensus on the nature of the graph schema, graph comprehension theories, coupled with support from other research domains, lead to two distinct hypotheses: the perceptual feature view and the invariant structure view. The perceptual feature view suggests that the surface-level features and the distinct perceptual properties of a graph determine the schema (Lohse, 1993). In Lohse's model of graph interpretation, each unique type of graph instantiates a different schema and, consequently, a different set of procedures for interpreting the graph. For example, bar graphs, line graphs, and pie charts would each activate a different schema. The scene-perception literature, which shares many characteristics with that of graph comprehension regarding time scale and the process of matching a visual array to a stored mental representation (Potter, 1993), also provides support for this view. Theories of scene perception suggest that perceptual characteristics, such as the identity of select key objects, may drive activation of the appropriate schema (Henderson \& Hollingworth, 1999). The most visible and distinguishing perceptual characteristic of a graph is the graphical pattern (Kosslyn, 1989); thus,

R. M. Ratwani, rratwani@gmu.edu 
this pattern may be the underlying feature that defines the graph schema. The graphical pattern is the pictorial object of the graph-for example, the actual lines in a line graph or the actual bars in a bar graph.

The invariant structure view suggests that certain broad categories, or types of graphs, may rely on a shared schema; however, the defining features that determine which graph types rely on the same schema are not clear. Pinker (1990) suggested that graph schemas are organized hierarchically; there is a general graph schema, which contains the common properties of all graphs, and there are graph-specific schemas for commonly encountered graph types. Peebles and Cheng $(2001,2003)$ have suggested that certain graphs that share similar features may rely on a similar graph schema; however, Peebles and Cheng $(2001,2003)$ did not specify those features. The notion that certain graph types share similar schemas, based on a common set of properties, is similar to the problem-type schemata in the problemsolving literature (Bernardo, 1994). Thus, a common invariant structure among certain graph types may be the underlying principle that determines the graph schema.

We suggest that the graphical framework may be the common invariant structure. The graphical framework is the structural component of a graph that often represents the basic concepts and operations for extracting information from the graph (Kosslyn, 1989). For example, in the line graph, bar graph, and horizontal bar graph in Fig- ure 1 , the framework is represented by the $x$ - and $y$-axes. These axes represent the Cartesian coordinate system on which these graphs are based. Similarly, in the pie chart and doughnut graph, the circle encompassing each graph is the framework. This structure represents the polar coordinate system on which these graphs are based. This framework may be the critical structural feature that determines the schema. Line and bar graphs may have different graphical patterns, but they share the same Cartesian coordinate framework and may share some underlying mental representations. Likewise, polar-coordinate-based graphs may rely on a common mental representation.

\section{The Mixing-Costs Paradigm}

In order to distinguish between the perceptual feature and invariant structure views, different graphs were examined in a mixing-costs paradigm (Los, 1996). This paradigm consists of presenting stimuli (graphs, in this case) in both pure and mixed blocks and then comparing reaction times (RTs) across the different blocks. An RT difference between the pure and mixed blocks can be attributed to a mechanistic difference in processing the stimuli. In this study, we attempted to isolate the activation of the graph schema as the only difference between stimuli.

To examine the graph schema, we held all of the stages of processing for each graph constant, except for the activation of the graph schema. Early visual processing and

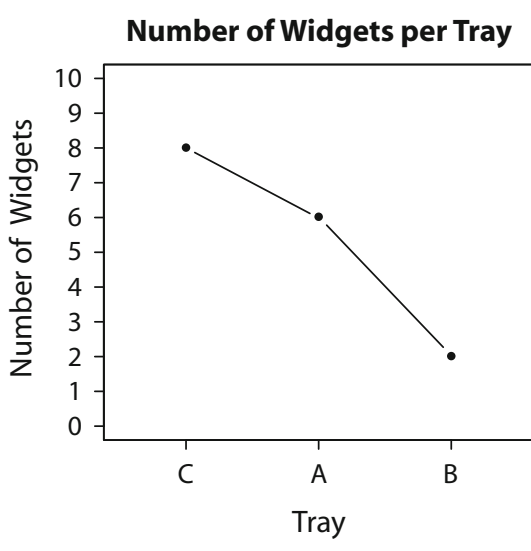

Number of Widgets per Tray

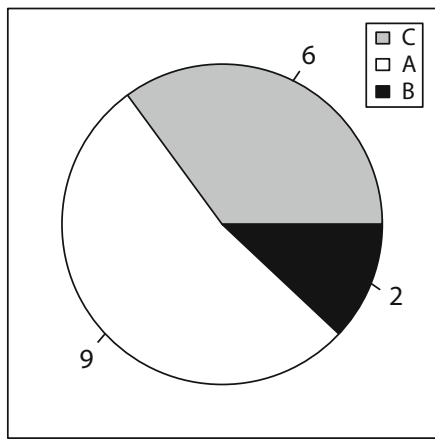

Number of Widgets per Tray

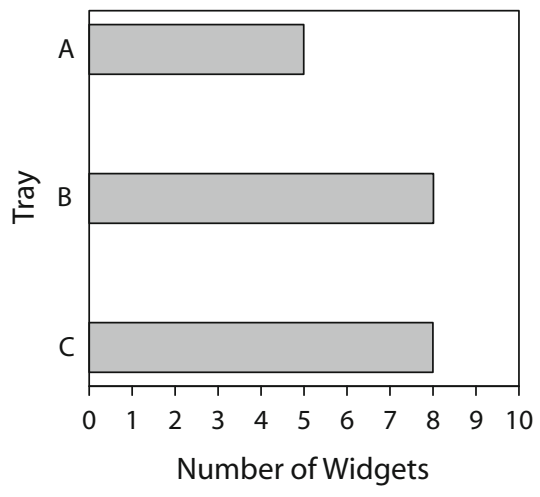

Number of Widgets per Tray

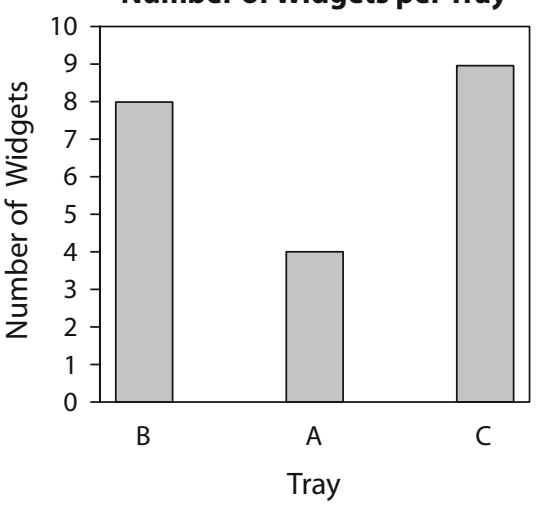

Number of Widgets per Tray

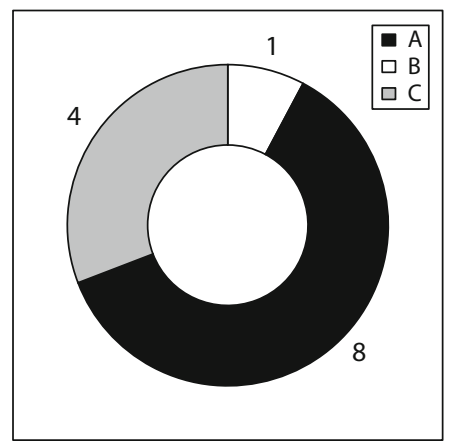

Figure 1. Examples of graph types used in Experiments 1-3. Upper left, line graph; upper right, bar graph; lower left, pie chart; lower right, doughnut graph; center, horizontal bar graph. 
the construction of the propositional representation of the graphs (i.e., Steps 1-2 from the graph comprehension theories) were equated by examining the same graph type across the pure and mixed blocks. Although these processes may vary slightly depending on the orientation of the graphical pattern, these differences should not have impacted mean RT. The conceptual question (Step 4) was equated for all graphs, by asking the same question for each graph, and, although the conceptual message (Step 5) might differ slightly from graph to graph, RT differences should be minimal. Thus, the activation of the graph schema was the only step that might vary, depending on the graph being examined, and the cost of activating the appropriate schema should be apparent using the mixing-costs paradigm.

Five graph types were examined: line graphs, bar graphs, pie charts, doughnut graphs, and horizontal bar graphs. Two graphs were examined at a time, in order to determine what characteristics underlie the graph schema. If a given set of graphs rely on different schemas, it should take more time to load the appropriate schema than it would if they activate the same schema, which should result in a time cost. In the mixed block, if the two graphs rely on different schema, the appropriate schema needs to be activated, resulting in longer RTs than in the pure block, where a new schema does not have to be activated (because it was activated on previous trials). If, however, the graphs being examined rely on a similar schema, the appropriate schema is already activated in the mixed block, just as it is in the pure block, resulting in no time cost. According to the perceptual feature view, pairs of graphs with unique graphical patterns should rely on different schemas, which should, therefore, result in time costs. The invariant structure view suggests that graphs with a common graphical framework will share similar schemas, which should result in no time costs.

\section{GENERAL METHOD}

\section{Participants}

Participants in each experiment were 18-31 undergraduate students (see Table 1 for the number of participants in each experiment). The participants were primarily first- or second-year psychology students participating for course credit.

\section{Materials}

Two graph types were tested in each experiment, which contained 80 samples of each graph type, resulting in a total of 160 graphs per experiment. Each randomly generated graph depicted the number of "Widgets" located in three different trays (A, B, and C), ranging from 1-9. In the line and bar graphs, the $x$-axis contained labels for the three different trays, and the $y$-axis was a scale ranging from 0 to 10 ; in the horizontal bar graphs, the axes were reversed. The order of tray labels was randomized for every graph. The doughnut graphs and pie charts contained a legend that assigned each tray (A, B, or C) to a specific colored segment (black, white, or gray), and a number between 1 and 9 was assigned to each segment in order to indicate the number of widgets that it represented. Examples of each of the graph types can be found in Figure 1. The color-tray association was randomized in every graph. To hold the conceptual question constant, participants were asked, "How many Widgets are there in Tray B?" for each graph. This type of extraction question was used because most of the theories of graph comprehension have been based on this type of question (Lohse, 1993; Pinker, 1990). In addition to the graphs, 80 text sentences (e.g., There were five ships in the bay.), for which the participants were required to enter the number appearing in the sentence, served as a filler task.

\section{Design}

In each experiment, two different graph types and text were presented in six different blocks; each block contained 40 stimuli. There were three pure blocks, with all the same stimuli type-two pure graph blocks and one pure text block - and three mixed blocks. One mixed block contained 20 of each graph type; the other two contained 20 of each graph type and 20 sentences. The stimuli in each block were randomly ordered. The three blocks with text were filler blocks and were not analyzed, resulting in three blocks of interest: two pure graph blocks and one mixed graph block. For example, in Experiment 1A, where line and bar graphs were examined, the blocks of interest were a pure block of line graphs, a pure block of pie charts, and a mixed block of line graphs and pie charts.

\section{Procedure}

Blocks were presented using a Latin square design. The stimuli were presented on a computer screen and RT data were collected. Participants were asked to respond to each stimulus as quickly and accurately as possible using the keypad. After a response, the next stimulus appeared immediately. Three practice trials were completed prior to each block.

\section{Data Analysis}

The two pure blocks of graphs were used to calculate two means, by averaging the RTs for all 40 stimuli in each block. The mixed block of graphs was segmented by trial type and graph type, yielding switch trials and nonswitch trials (Los, 1999). The switch trials consisted of a particular kind of graph preceded by a different kind of graph (e.g., a line graph preceded by a pie chart), whereas the nonswitch trials consisted of a particular graph preceded by the same kind of graph (e.g., a line graph preceded by a line graph). For example, in Experiment $1 \mathrm{~A}$, where line graphs and pie charts were examined, analysis of the pure blocks resulted in a mean for line graphs and one for pie charts. Analysis of the mixed block resulted in four means: switchline, switch-pie, nonswitch-line, and nonswitch-pie.

The pure block RT means reflect a highly activated schema for the particular graph being viewed, since the same kind of graph was

Table 1

Average Pure, Switch, and Nonswitch Reaction Times (RTs, in Milliseconds) for Experiments 1-3

\begin{tabular}{|c|c|c|c|c|c|c|}
\hline \multirow[b]{3}{*}{ Graph Type } & \multirow{2}{*}{\multicolumn{2}{|c|}{$\begin{array}{c}\text { Pure } \\
\text { Block RT }\end{array}$}} & \multicolumn{4}{|c|}{ Mixed Block RT } \\
\hline & & & \multicolumn{2}{|c|}{ Switch } & \multicolumn{2}{|c|}{ Nonswitch } \\
\hline & $M$ & $S D$ & $M$ & $S D$ & $M$ & $S D$ \\
\hline \multicolumn{7}{|c|}{ Experiment 1A $(N=20)$} \\
\hline Line & 2,010 & 291 & 2,136 & 275 & 2,043 & 359 \\
\hline Pie chart & 2,227 & 322 & 2,346 & 296 & 2,233 & 290 \\
\hline \multicolumn{7}{|c|}{ Experiment 1B $(N=22)$} \\
\hline Bar & 1,798 & 222 & 1,914 & 305 & 1,876 & 235 \\
\hline Pie chart & 2,092 & 235 & 2,270 & 325 & 2,177 & 309 \\
\hline \multicolumn{7}{|c|}{ Experiment $1 \mathrm{C}(N=21)$} \\
\hline Line & 1,916 & 324 & 1,941 & 302 & 1,890 & 255 \\
\hline Bar & 1,775 & 278 & 1,780 & 282 & 1,770 & 274 \\
\hline \multicolumn{7}{|c|}{ Experiment $2 \mathrm{~A}(N=31)$} \\
\hline Line & 1,971 & 302 & 2,087 & 307 & 1,997 & 357 \\
\hline Doughnut & 2,176 & 246 & 2,268 & 301 & 2,183 & 296 \\
\hline \multicolumn{7}{|c|}{ Experiment $2 \mathrm{~B}(N=23)$} \\
\hline Bar & 1,871 & 218 & 2,010 & 265 & 1,893 & 240 \\
\hline Doughnut & 2,247 & 289 & 2,395 & 306 & 2,269 & 300 \\
\hline \multicolumn{7}{|c|}{ Experiment $2 \mathrm{C}(N=21)$} \\
\hline Pie chart & 2,293 & 343 & 2,308 & 337 & 2,237 & 357 \\
\hline Doughnut & 2,440 & 343 & 2,373 & 323 & 2,293 & 301 \\
\hline \multicolumn{7}{|c|}{ Experiment $3(N=18)$} \\
\hline Line & 1,959 & 247 & 1,981 & 278 & 1,985 & 267 \\
\hline Horizontal bar & 1,876 & 231 & 1,874 & 254 & 1,818 & 247 \\
\hline
\end{tabular}


Table 2

Time Costs (in Milliseconds) for the Pairs of Graph Types Examined in Experiments 1-3

\begin{tabular}{lccccc}
\hline & \multicolumn{4}{c}{ Pie } & Horizontal \\
& Line & Bar & Charts & Doughnut & Bar \\
\hline Line & - & 5 & $\mathbf{1 1 9}$ & $\mathbf{9 2}$ & -2 \\
Bar & 25 & - & $\mathbf{1 7 8}$ & $\mathbf{1 4 8}$ & - \\
Pie charts & $\mathbf{1 2 6}$ & $\mathbf{1 1 6}$ & - & -67 & - \\
Doughnut & $\mathbf{1 1 6}$ & $\mathbf{1 3 9}$ & 15 & - & - \\
Horizontal bar & 22 & - & - & - & -
\end{tabular}

Note-Bold cells represent a significant time cost. Each column of numbers represents the time cost to that particular graph when it is mixed with each of the other types of graph. For example, the cost to line graphs when mixed with bar graphs was $25 \mathrm{msec}$ (n.s.), and the cost to line graphs when mixed with pie charts was $126 \mathrm{msec}$.

seen throughout the block. The mixed block switch RT means reflect the process of having to activate the appropriate mental representation required to process the particular graph being viewed, since the previously viewed graph was of a different type. Thus, the switch RT means capture the time cost of activating the appropriate schema. The nonswitch RT means reflect a more activated schema, as compared with the switch RT means, since the previously viewed graph was of the same type as the current graph. For the purposes of this study, we focused on the pure means and switch means, since we wanted to compare highly activated schemas with situations where the schema needed to be activated. This way of calculating time costs is different from that in the task-switching literature (Monsell, 2003). ${ }^{1}$ Table 1 shows the pure, switch, and nonswitch means for all experiments.

In each experiment, the graph and block type were compared using a two-way ANOVA. A main effect of graph type reflects differences in the time required to process a particular graph. An effect of block type reflects the time costs between the pure and mixed blocks. There were no significant interactions in any of the experiments. Incorrect responses and RTs greater than three standard deviations from the mean (less than $5 \%$ of the data) were excluded. Table 2 shows the actual time costs for pairs of graphs across all experiments.

\section{EXPERIMENTS 1A-1C}

Line graphs, bar graphs, and pie charts were examined in the first set of experiments. Experiment $1 \mathrm{~A}$ compared line graphs with pie charts, and Experiment 1B compared bar graphs with pie charts. Both the perceptual feature and the invariant structure view suggest different mental representations for each experiment, since graph types with different patterns and frameworks (i.e., Cartesian vs. polar coordinates) were compared. Thus, there should be time costs, reflected as a significant block-type effect, in each experiment, as well as a significant graph-type effect.

In Experiment 1C, line and bar graphs were compared. The perceptual feature view suggests that there should be time costs and a significant block-type effect, whereas the invariant structure view suggests that there should be no time costs, since line and bar graphs share the same Cartesian framework. Because bar graphs are best for extracting discrete data (Zacks \& Tversky, 1999), their associated RTs should be faster than those for line graphs, reflected in a significant graph-type effect.

\section{Results}

Experiment 1A: Line graphs and pie charts. RTs for line graphs were significantly faster than those for pie charts $\left[F(1,19)=19.1, M S_{\mathrm{e}}=47,562, p<.001\right]$. The main effect of block type was significant $[F(1,19)=12.8$, $\left.M S_{\mathrm{e}}=23,459, p<.01\right]$; RTs for the mixed blocks were slower than those for the pure blocks.

Experiment 1B: Bar graphs and pie charts. RTs for bar graphs were significantly faster than those for pie charts $\left[F(1,21)=115.21, M S_{\mathrm{e}}=20,142, p<.001\right]$. The main effect of block type was significant $[F(1,21)=14.3$, $\left.M S_{\mathrm{e}}=33,256, p<.01\right] ; \mathrm{RT}$ for the mixed blocks were slower than those for the pure blocks.

Experiment 1C: Line and bar graphs. RTs for bar graphs were significantly faster than those for line graphs $\left[F(1,20)=38.02, M S_{\mathrm{e}}=12,532, p<.001\right]$. The main effect of block type, however, was not significant $[F(1,20)=$ $\left.0.15, M S_{\mathrm{e}}=30,391, p=.7\right]$; there was no difference between the RTs for the pure and mixed blocks. A power analysis showed that the probability of detecting an effect was greater than $85 \%$, which suggests that the null effect is not likely to have been an issue of power.

\section{Discussion}

In each experiment, a significant effect of graph type was found, suggesting a different overall processing time for each graph type. Furthermore, when the participants were given the task of extracting specific values, the rank ordering of processing times (i.e., RTs for bar graphs being the fastest, followed by those for line graphs, and then those for pie charts) was in agreement with the graph comprehension research, which suggests that bar graphs are best for extracting discrete values (Zacks \& Tversky, 1999).

The block-type effects in Experiments 1A and 1B suggest that both line and bar graphs may rely on different mental representations than do pie charts. In Experiment $1 \mathrm{C}$, when two Cartesian-coordinate graphs were examined, there were no time costs. Together, these results provide support for the invariant structure view and for the role of the graphical framework in determining the schema. In Experiment 2, we sought to replicate these findings with different types of graphs.

\section{EXPERIMENTS 2A-2C}

To further test the invariant structure view, doughnut graphs - a polar-coordinate-based graph type that relies on the same framework as pie charts-were examined. If the graphical framework determines the schema, doughnut graphs paired with line graphs (Experiment 2A) and bar graphs (Experiment 2B) should incur time costs, since these combinations pair graphs with different frameworks. However, doughnut graphs paired with pie charts (Experiment 2C) should not incur time costs, since they rely on the same framework.

\section{Results}

Experiment 2A: Line and doughnut graphs. RTs for line graphs were significantly faster than those for doughnut graphs $\left[F(1,30)=37.1, M S_{\mathrm{e}}=31,065, p<.01\right]$. The main effect of block type was significant $[F(1,30)=9.3$, $\left.M S_{\mathrm{e}}=36,178, p<.01\right] ; \mathrm{RTs}$ for the mixed blocks were slower than those for the pure blocks. 
Experiment 2B: Bar and doughnut graphs. RTs for bar graphs were significantly faster than those for doughnut graphs $\left[F(1,22)=61.8, M S_{\mathrm{e}}=53,795, p<.001\right]$. The main effect of block type was significant $[F(1,22)=15.3$, $\left.M S_{\mathrm{e}}=30,852, p<.01\right]$; RTs for the mixed blocks were slower than those for the pure blocks.

Experiment 2C: Pie charts and doughnut graphs. RTs for pie charts were significantly faster than those for doughnut graphs $\left[F(1,20)=9.1, M S_{\mathrm{e}}=26,090, p<.01\right]$. The main effect of block type was not significant $[F(1,20)=$ $\left.0.75, M S_{\mathrm{e}}=19,610, p=.4\right]$; there was no difference between the RTs for the pure blocks and those for mixed blocks. A power analysis showed that the probability of detecting an effect was greater than $85 \%$, which suggests that the null effect is not likely to have been an issue of power.

\section{Discussion}

The results of Experiment 2 further support the invariant structure view. In Experiments 2A and 2B, when polar- and Cartesian-coordinate graph types were examined in the same block, the results showed time costs. In Experiment 2C, when two polar-coordinate graph types were examined, there were no time costs. Similar to that of Experiment 1, the graph-type effect was significant in all cases, which suggests a difference in processing times for each graph type.

Although Experiments 1 and 2 provided support for the invariant structure view, one possible explanation for the null block-type effect in Experiments 1C and 2C was that these graph types required the same visual procedures for extracting information. For example, in Experiment 1C, participants may have realized that they could look to the $x$-axis to find the desired tray, then scan up and over to the $y$-axis, regardless of the graph type presented. Perhaps the absence of a block-type effect was due to the same visual procedures' being used and not to a shared mental representation. Although the consistent significant graph-type effects in each experiment provide some evidence against this argument, Experiment 3 specifically examined this issue.

\section{EXPERIMENT 3}

To examine whether the null block-type effect in Experiments $1 \mathrm{C}$ and $2 \mathrm{C}$ was due to a reliance on the same visual procedures, graph types that shared a framework but required different visual procedures were tested. Line graphs and horizontal bar graphs share the same graphical framework; however, a different set of visual procedures is required for extracting information. Horizontal bar graphs require one to first look to the $y$-axis, as opposed to the $x$-axis in line graphs. If the graphical framework is the underlying structural component that determines the schema, there should be no time costs.

\section{Results and Discussion}

RTs for horizontal bar graphs were faster than those for line graphs $\left[F(1,17)=15.9, M S_{\mathrm{e}}=10,784, p<\right.$ $.001]$. The main effect of block type was not significant $\left[F(1,17)=0.08, M S_{\mathrm{e}}=25,634, p=.8\right]$; there was no difference between the RTs for the pure blocks and those for the mixed blocks. A power analysis showed that the probability of detecting an effect was greater than $85 \%$, which suggests the null effect is not likely to have been an issue of power.

The main effect of graph type suggests different processing times for each graph type. No time costs were incurred in this experiment, despite the fact that different visual procedures were required for extracting information from the graphs. This suggests that using the same visual procedures does not account for the null effects observed in the previous experiments; we believe that the activation of the graph schema (or lack thereof) accounts for the block-type effects found in those experiments. The results of this experiment provide further evidence for the invariant structure view; the line and horizontal bar graphs share a common framework and, consequently, rely on a similar mental representation.

\section{GENERAL DISCUSSION}

Using the mixing-costs paradigm, we systematically examined the RTs for different graph types, and we have shown that the graph schema seems to be based on an invariant structure shared by certain categories of graphs. Specifically, the graphical framework determines the schema; graph types that share the same framework rely on a similar mental representation. The framework represents the conceptual knowledge necessary for extracting information from the graph. We argue that once the graphical framework for a particular graph is perceptually identified, it is matched to the mental representation for the particular graph type (e.g., bar graphs are matched to the Cartesian-coordinate framework), thus activating the concepts and operations required for extracting information.

Our view of the graph schema is not far from those of Pinker (1990) and Peebles and Cheng (2001). Pinker suggested a hierarchical organization to the graph schema, and our data align with that view. Our results suggest that a critical component of this hierarchy is the graphical framework. Furthermore, we have clearly specified the Peebles and Cheng (2001) description of the graph schema by illustrating that the framework determines the schema.

Our results provide a foundation for understanding graph schemas; however, one of the limitations of this study is that the same specific extraction question was asked in each experiment. Although this was purposefully done to isolate the activation of the graph schema, the role of schemas in more complex graphs with more complex questions requires further investigation. The mixing-costs methodology proved to be a powerful technique in isolating the activation of the graph schema in these experiments; a similar methodology can be used to extend research on graph schemas and to investigate mental representations in other domains.

An obvious question that arises is how people are able to comprehend novel graphs for which they do not have a stored mental representation. We believe that two processes are at work in the comprehension of novel graphs. Upon examination of the graph, partial matching between the early visual array and a stored mental representation 
may occur. This partial matching will activate a set of operations that may be applicable to the graph. In addition to a partial matching mechanism, similarities in relational structure between concepts and the novel graph are likely to play a role in interpretation of the graph (Gattis, 2002).

\section{AUTHOR NOTE}

This work was supported by ONR Grant N0001403WX30001. The authors thank Meredith Gattis, Chris Kello, Matt Peterson, Barbara Spellman, and Jeff Zacks for their comments and suggestions. Correspondence concerning this article should be addressed to R. M. Ratwani, Department of Psychology, George Mason University, Fairfax, VA 22030 (e-mail: rratwani@gmu.edu).

\section{REFERENCES}

Bernardo, A. B. I. (1994). Problem-specific information and the development of problem-type schemata. Journal of Experimental Psychology: Learning, Memory, \& Cognition, 20, 379-395.

Gattis, M. (2002). Structure mapping in spatial reasoning. Cognitive Development, 17, 1157-1183.

Henderson, J. M., \& Hollingworth, A. (1999). High-level scene perception. Annual Review of Psychology, 50, 243-271.

Kosslyn, S. M. (1989). Understanding charts and graphs. Applied Cognitive Psychology, 3, 185-225.

Lewandowsky, S., \& Behrens, J. T. (1999). Statistical graphs and maps. In F. T. Durso, R. S. Nickerson, R. W. Schvaneveldt, S. T. Dumais, D. S. Lindsay, \& M. T. H. Chi (Eds.), Handbook of applied cognition (pp. 513-549). Chichester, U.K.: Wiley.

LoHse, G. L. (1993). A cognitive model for understanding graphical perception. Human-Computer Interaction, 8, 353-388.

Los, S. A. (1996). On the origin of mixing costs: Exploring information processing in pure and mixed blocks of trials. Acta Psychologica, 94, $145-188$.

Los, S. A. (1999). Identifying stimuli of different perceptual categories in mixed blocks of trials: Evidence for cost in switching between computational processes. Journal of Experimental Psychology: Human Perception \& Performance, 25, 3-23.

Monsell, S. (2003). Task switching. Trends in Cognitive Sciences, 7, 134-140.

Peebles, D., \& Cheng, P. C.-H. (2001). Graph-based reasoning: From task analysis to cognitive explanation. In J. D. Moore \& K. Stenning (Eds.), Proceedings of the Twenty-Third Annual Conference of the Cognitive Science Society (pp. 762-767). Mahwah, NJ: Erlbaum.

Peebles, D., \& Cheng, P. C.-H. (2003). Modeling the effect of task and graphical representation on response latency in a graph reading task. Human Factors, 45, 28-46.

PinKer, S. (1990). A theory of graph comprehension. In R. Freedle (Ed.), Artificial intelligence and the future of testing (pp. 73-126). Hillsdale, NJ: Erlbaum.

PotTer, M. C. (1993). Very short-term conceptual memory. Memory \& Cognition, 21, 156-161.

Shah, P., \& Carpenter, P. A. (1995). Conceptual limitations in comprehending line graphs. Journal of Experimental Psychology: General, 124, 43-61.

SimKIN, D. K., \& Hastie, R. (1987). An information processing analysis of graph perception. Journal of the American Statistical Association, 82, 454-465.

ZACKS, J., \& TVERSKY, B. (1999). Bars and lines: A study of graphic communication. Memory \& Cognition, 27, 1073-1079.

\section{NOTE}

1. In the task-switching literature (Monsell, 2003), mixing costs are calculated by taking the difference between pure and nonswitch means; we have used pure and switch means. We have run our analyses both ways, and the results are consistent in all but one case.

(Manuscript received March 9, 2007; revision accepted for publication February 8, 2008.) 